\title{
Conserved DNA motifs in the type II-A CRISPR leader region
}

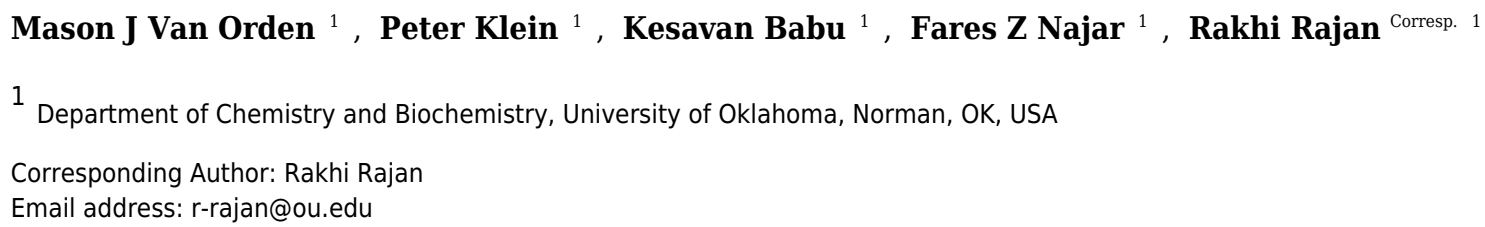

The CRISPR-Cas systems consist of RNA-protein complexes that provide bacteria and archaea with sequence-specific immunity against bacteriophages, plasmids, and other mobile genetic elements. Bacteria and archaea become immune to phage or plasmid infections by inserting short pieces of the intruder DNA (spacer) site-specifically into the leader-repeat junction in a process called adaptation. Previous studies have shown that parts of the leader region, especially the 3 ' end of the leader, are indispensable for adaptation. However, a comprehensive analysis of leader ends remains absent. Here, we have analyzed the leader, repeat, and Cas proteins from 167 type II-A CRISPR loci. Our results indicate two distinct conserved DNA motifs at the 3' leader end; ATTTGAG (noted previously in the CRISPR1 locus of Streptococcus thermophilus DGCC7710) and a newly defined CTRCGAG, associated with the CRISPR3 locus of $S$. thermophilus DGCC7710. A third group with a very short CG DNA conservation at the 3 ' leader end is observed mostly in Lactobacilli. Analysis of the repeats and Cas proteins revealed clustering of these CRISPR components that mirrors the leader motif clustering, in agreement with the coevolution of CRISPR-Cas components. Based on our analysis of the type II-A CRISPR loci, we implicate leader end sequences that could confer site-specificity for the adaptationmachinery in the different subsets of type II-A CRISPR loci. 
1

2 Conserved DNA motifs in the type II-A CRISPR leader region

3

4

5

6

7

8 Mason J. Van Orden ${ }^{1 \#}$, Peter Klein ${ }^{1 \#}$, Kesavan Babu ${ }^{1}$, Fares Z. Najar ${ }^{1}$, and Rakhi Rajan ${ }^{1 *}$

9

10

11

12 'Department of Chemistry and Biochemistry, University of Oklahoma, Norman, OK, USA

13 \# Equal first authors

14

15

$16{ }^{*}$ Correspondence: r-rajan@ou.edu 


\section{Abstract}

18 The CRISPR-Cas systems consist of RNA-protein complexes that provide bacteria and archaea 19 with sequence-specific immunity against bacteriophages, plasmids, and other mobile genetic 20 elements. Bacteria and archaea become immune to phage or plasmid infections by inserting short 21 pieces of the intruder DNA (spacer) site-specifically into the leader-repeat junction in a process 22 called adaptation. Previous studies have shown that parts of the leader region, especially the 3' 23 end of the leader, are indispensable for adaptation. However, a comprehensive analysis of leader 24 ends remains absent. Here, we have analyzed the leader, repeat, and Cas proteins from 167 type 25 II-A CRISPR loci. Our results indicate two distinct conserved DNA motifs at the 3' leader end; 26 ATTTGAG (noted previously in the CRISPR1 locus of Streptococcus thermophilus DGCC7710) 27 and a newly defined CTRCGAG, associated with the CRISPR3 locus of S. thermophilus 28 DGCC7710. A third group with a very short CG DNA conservation at the 3' leader end is observed 29 mostly in Lactobacilli. Analysis of the repeats and Cas proteins revealed clustering of these 30 CRISPR components that mirrors the leader motif clustering, in agreement with the coevolution 31 of CRISPR-Cas components. Based on our analysis of the type II-A CRISPR loci, we implicate 32 leader end sequences that could confer site-specificity for the adaptation-machinery in the 33 different subsets of type II-A CRISPR loci. 


\section{Introduction}

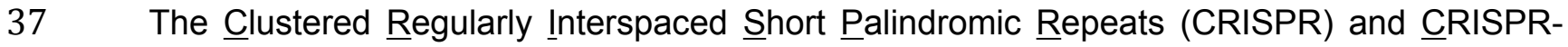

38 associated (Cas) proteins constitute an RNA-based adaptive immune system that protects 39 bacteria and archaea against phages and mobile genetic elements ${ }^{1-5}$. CRISPR-Cas systems 40 inactivate intruder DNA, RNA, or both based on the sequence similarity of small CRISPR RNAs 41 (crRNAs) to the invading genetic element and thus protect microbes from phage infections and 42 horizontal gene transfer ${ }^{2,6-11}$. The CRISPR-Cas systems are classified into six types (Ito VI) with 43 several subtypes within each type based on Cas protein composition ${ }^{11-13}$. An individual bacterium can have multiple CRISPR loci belonging to different CRISPR types. Though types I and III share certain similarities in the overall mechanism of action including crRNA association with multiple Cas proteins ${ }^{14-18}$, types II, V, and VI use a single multi-domain protein (Cas9, Cpf1, or C2c2 respectively) along with cognate RNA components for activity ${ }^{11} 19-22$. Cas9 along with a guide RNA is widely being used for genome editing applications, and is being pursued for gene therapy and gene regulation applications ${ }^{23,24}$.

The CRISPR genomic locus is present in either the chromosomal or plasmid DNA as a recurring array of "repeat" and "spacer" units, both of which usually range from approximately 24 to 50 nucleotides $^{2,3,25,26}$. Repeats consist of palindromic DNA sequences, while spacers are derived from invader genetic material, and experimental evidence supports the role of spacers in conferring sequence-specific resistance against bacteriophages ${ }^{4,9}$ and plasmids ${ }^{8}$. The cas genes that code for the CRISPR system's essential protein components are often located close to the CRISPR locus ${ }^{26,27}$. The CRISPR leader located 5' to the first repeat consists of an A/T-rich region around 100-500 nucleotides long with embedded transcriptional promoters ${ }^{28-30}$. In certain CRISPR types, a 3-5 nucleotide long region present in the invading DNA (Protospacer Adjacent

59 Motif (PAM)) is crucial for Cas proteins to differentiate self from non-self DNA ${ }^{2,31,32}$, where as in 60 other types this is defined by the crRNA-DNA base pairing patterns ${ }^{7}$. 
61 There are three stages in CRISPR mediated defense: adaptation (acquiring new spacers), crRNA

62 biogenesis, and interference ${ }^{2,33}$. The adaptation process differs between CRISPR types. The 63 proteins, Cas1 and Cas2, are universally present and essential for adaptation in most of the 64 CRISPR types ${ }^{30,34}$. In certain type II subtypes, Csn2 and Cas4 are also implicated in adaptation 65 along with Cas1 and Cas2 ${ }^{4,35}$. The new spacers are inserted at the leader-repeat junction in most 66 systems, although some variability have been observed in Sulfolobus solfataricus ${ }^{36}$. A minimum 67 of one repeat along with the leader region can promote spacer insertion and in certain CRISPR 68 subtypes, one of the strands of the double-stranded intruding DNA is preferred for spacer 69 acquisition ${ }^{29,37}$. A new spacer insertion is always accompanied by repeat duplication and the first 70 repeat serves as a template for new repeat synthesis ${ }^{30}$. Even though recognition of the PAM 71 sequence by Cas 9 is essential for acquisition and insertion of spacers in the correct orientation in 72 vivo in type II CRISPR systems ${ }^{38-40}$, it was recently demonstrated that Cas1 and Cas2 from 73 Streptococcus pyogenes can specifically integrate spacers into the leader-repeat junction based 74 solely on intrinsic sequence specificity of the first repeat ${ }^{41}$.

75 Streptococcus thermophilus (Sth) DGCC7710 is a model organism widely used for studying various CRISPR processes. Sth DGCC7710 has a total of four CRISPR loci in its genome, and loci CRISPR1 and CRISPR3 belong to type II-A ${ }^{42-44}$. It was experimentally shown that in Sth DGCC7710, both CRISPR1 and CRISPR3 are active in acquiring new spacers in relation to a new phage or plasmid threat, with CRISPR1 being more active due to the higher frequency of new spacer insertion in this locus compared to CRISPR34,29,35,40,42,44-47. A phylogenetic analysis showed that the repeat and cas genes segregate specifically with the locus type (CRISPR1 vs CRISPR2 vs CRISP3) in streptococci and in several bacteria belonging to different genera ${ }^{46}$.

83 Several studies pointed to the indispensability of the 3' end of the leader in CRISPR 84 adaptation $29,30,36,48-53$. In the CRISPR1 locus of Sth DGCC7710 (type II-A), a cis-acting element at 85 the 3' end of the leader (ATTTGAG) was shown to be essential for adaptation and this region is 86 conserved in several type II-A systems ${ }^{29}$. In Escherichia coli BL21 strain, a type I-E CRISPR locus 
87 showed defective adaptation following deletions or mutation in the 60 nucleotides towards the 3' 88 end of the CRISPR leader ${ }^{30}$. A pair of inverted repeat regions in the first repeat along with the 89 leader end sequence is critical for adaptation in the type IB system of Haloarcula hispanica ${ }^{54}$. 90 Recently, a study in type I CRISPR systems identified leader DNA sequences that are specifically 91 recognized by the integration host factor (IHF) protein to facilitate leader-proximal spacer 92 integration under in vitro conditions. ${ }^{55}$ It was later shown, however, that under in vitro conditions 93 type II systems integrate spacers specifically into the leader-proximal regions by Cas1-Cas2 94 activity alone, without the participation of another protein. ${ }^{41}$ This highlights the differences in the 95 mechanism of spacer integration between CRISPR types and the possibility of divergent 96 contributions of the leader and repeat sequences in type-specific adaptation.

98 In order to identify the leader and repeat DNA sequence conservations that may contribute to 99 site-specific spacer integration in type II-A CRISPR systems, we report the analysis of the leaderrepeat region belonging to 167 type II-A CRISPR loci from 50 different genera. 87 of the 167 loci have the 3' leader end conserved as ATTTGAG (Group 1), 55/167 loci have their 3' leader end conserved as CTRCGAG (Group 2), and 25/167 possess a CG conservation at the 3' end of the leader (Group 3). Previous studies that established the importance of ATTTGAG and ACGAG leader end sequences in adaptation of Sth DGCC7710 and S. pyogenes ${ }^{29}$, respectively, point to the functional significance of the conserved DNA motifs. A detailed analysis of the Cas proteins associated with the 167 type II-A loci shows protein sequence specificities that delineate these proteins into groups that mirror the leader end conservation. Thus, our study establishes distinct sub-group specific DNA sequence conservation patterns in the type II-A CRISPR leader that extends across many diverse bacteria demonstrating the ubiquitous nature of the 3'-leader end conservations that were previously observed only in related streptococcal species. 


\section{Methods}

\section{Processing of genomic data}

114 In this study, the type II-A loci were collected by multiple ways. Initially, Bacterial Generic Feature

115 Format (GFF) and accessioned protein product FASTA files were downloaded from NCBI and 116 scanned for II-A specific Cas protein names (Cas9/Csn1 and Csn2) in the annotation field. The 117 genomes containing Cas9/Csn1 and/or Csn2 annotation entries were downloaded from NCBI in 118 GenBank format. The datasets were screened manually for the presence of $\operatorname{cas} 1, \operatorname{cas} 2, \operatorname{cas} 9$, 119 and csn2, and only the loci with all four type II-A specific cas genes were used for further analysis.

120 The genomic region flanking downstream of the csn2 gene was further processed to extract the 121 leader and the first repeat of the CRISPR array. The protein sequences of Cas9, Cas1, Cas2, 122 and Csn2 that were coded by the upstream region flanking the csn2 gene were extracted from $123 \mathrm{NCBI}$. The presence of all four proteins limits our dataset to strictly type II-A loci. A total of 129 124 loci were identified based on Cas9/Csn1 and/or Csn2 annotation search. Previously, Chylinski et 125 al reported type II-A loci based on a Cas9 sequence search ${ }^{56,57}$. A total of 32 type II-A loci that 126 represented species and genera that were absent in our initial dataset were selected for our study. 127 In addition, we performed protein sequence homology search by DELTA-BLAST ${ }^{58}$ using a 128 representative Csn2 sequence from each subfamily as mentioned in Chylinski et al $2014^{56}$ ((NCBI 129 protein accession number: 116101487 for subfamily I, 116100822 for subfamily II, 389815356 for 130 subfamily III, 385326557 for subfamily IV, 315659845 for subfamily V as mentioned in Chylinski 131 et al 2014)56. By this search a total of 6 loci were identified from bacterial genera Weissella, 132 Globicatella, Nosocomiicoccus, Caryophanon and Virgibacillus. The final dataset consisted of 133167 type II-A loci with a wide representation based on the current knowledge of type II-A diversity.

134 A total of 50 different bacterial genera were present in our dataset. (Table S1 and S2).

135 The orientation of the Cas proteins was used in assessing the transcription direction of the leader136 repeat units. To analyze leader and repeat sequences, an approximately 400-nucleotide stretch 
137 of sequences downstream of csn2 gene were examined using CRISPR finder tool ${ }^{59}$, and an in-

138 house script to locate the tandem repeats. Since there were differences in the repeat length as it

139 exists in the genomic locus and as reported in the CRISPRdb ${ }^{59}$, we used the in-house program

140 to locate the repeats (Table S3). The accuracy of the repeat extracted by our script was validated

141 manually by checking the genomic data for the length and sequence of the repeat within a

142 CRISPR array. The loci that lacked predicted repeats or Cas protein(s) were omitted from further

143 analysis. In the case of bacteria with multiple CRISPR types, the components belonging to a

144 particular type II-A locus were taken as one dataset. For example, Sth DGCC7710 has four

145 CRISPR loci. Only loci 1 and 3 that correspond to type II-A were selected for our analysis. The

146 Cas proteins and leader-repeat elements of CRISPR1 were kept as one unit, while that belonging

147 to CRISPR3 represented another unit. Recently, several bioinformatics tools for the identification

148 and analysis of leader and repeat regions have been developed ${ }^{60,61}$. For a selected subset, we

149 compared the orientation of leader sequences and repeats as predicted by CRISPRDetect tool ${ }^{61}$

150 and our results, and saw agreement between the methods.

151 Sequence Alignment

152 We used MUSCLE with its default settings ${ }^{62}$ to perform all the sequence alignments in this study.

153 The MUSCLE output was used to generate phylogenetic trees with MEGA6 ${ }^{63}$ using the Maximum

154 Likelihood Tree option and Jones-Taylor-Thornton (JTT) model. Additionally, MUSCLE 155 alignments were used to generate alignment figures in UGENE ${ }^{64}$ and sequence logos with 156 WebLogo ${ }^{65}$.

157

\section{Results}

159 Analysis of the 3' end of the leader

160 An initial sequence alignment of the last 20 nucleotides of the leader plus the first repeat showed

161 that the 167 loci clustered into distinct groups. These groups had recognizable conservation at 
162 the last 7 nucleotides of the 3 ' end of the leader and the first 4 nucleotides of the 5 ' end of the first

163 repeat, or the leader-repeat junction. To obtain an unbiased separation of the different groups, a

164 Cas1 phylogenetic tree was constructed based on protein sequence similarity. The loci belonging

165 to the different clades of the Cas1 tree were grouped together and a sequence alignment of the

166 last 20 nucleotides of the leader along with the first repeat was performed. In order to facilitate

167 interpretation of the trees and alignments, a smaller representative sample of 62 loci was used to

168 generate the main figures and show the relevant relationships. Figures incorporating all of the

169167 loci can be found in the supplementary data. Each of the 3 groups was aligned separately to

170 discern the level of conservation within each group (Fig. 1 and 2, Fig. S1). Strict conservation is

171 seen at the 3' end of the leader as well as at the 5' end of the repeat. Group 1 has the 3' leader

172 end conserved as ATTTGAG (Fig. 1) and Group 2 has the 3'-leader end conserved as CTRCGAG

173 (where R represents a purine) (Fig. 2a). Group 3 has a shorter two nucleotide conservation of CG

174 at the 3' leader end. In Groups 1 and 2, the last three nucleotides are conserved as GAG (Fig.

175 2b). An A-rich region is partially conserved adjacent to the CG leader end of Group 3. Interestingly,

176 the CRISPR1 locus of Sth DGCC7710 has the 3' leader end conserved as ATTTGAG while the

177 CRISPR3 locus has the 3' leader end conserved as CTACGAG. Of the type II-A CRISPR loci

178 analyzed, 87 belonged to Group1, 55 belonged to Group 2, and 25 belonged to Group 3. Out of

179 the 50 genera analyzed, Group 2 consists of only 5 genera (Streptococcus, Enterococcus,

180 Listeria, Lactobacillus and Weissella) while Group 1 is much more diverse with 42 different

181 genera. Group 3 accounts for 7 genera, but has many loci belonging to the Order Lactobacillales.

182 The leader-repeat junction of Groups 1 and 2 is conserved as GAG/GTTT while in Group 3 it is 183 weakly conserved as CG/GTTT.

184

\section{Analysis of the repeat region}

186 The length of the repeat for the type II-A loci analyzed was 36 nucleotides except in 4 cases

187 (Enterococcus hirae ATCC 9790 (35 nucleotides long), Fusobacterium sp. 1_1_41FAA (37 
188 nucleotides long), Lactobacillus coryniformis subsp. coryniformis KCTC 3167 (37 nucleotides 189 long), and Lactobacillus sanfranciscensis TMW 1.1304 (35 nucleotides long)). The first repeat 190 sequences of the 3 groups didn't possess any distinguishable motifs that corresponded to the 191 segregation of the different groups (Fig. 3, Fig. S2). There is a strong sequence conservation at 192 the 5' end of the repeat as GTTT in all the type II-A loci analyzed (Fig. 3 and Fig. S2). Groups 1 193 and 2 also share a conserved AAAC motif at the 3' end of the repeat. Group 3 members have a 194 conserved C at the 3' end of the repeat, along with a less conserved A-rich region ahead of the 195 C. The repeat sequence belonging to the Group 2 loci is highly conserved across the entire length 196 of the repeat, which may be attributed to the limited number of genera (5) comprising this group 197 compared to Group 1 (42). In all the type II-A loci analyzed, the first and last nucleotides of the 198 first repeat are conserved as $G$ and $C$ respectively. A phylogenetic tree was generated using the 199 first repeat sequence of the type II-A loci (Fig. 3 and Fig. S2). Even though the reliability of 200 branching is low due to the short length of the sequence, the branches segregate such that 201 members within a clade have similar repeat and leader end conservations. Recently, it was 202 suggested that sequences at the 5' and 3' ends of the repeat in S. pyogenes type II-A system 203 could be the motifs recognized by Cas 1 during spacer acquisition. ${ }^{41}$ Hence, the conserved 5 ' and 204 3' repeat ends observed in the first two groups might indicate type II-A specific repeat ends that 205 are essential for adaptation. Further experimental studies will be required to analyze whether the 206 loosely conserved sequences at the 3' end of the repeat impact effective adaptation in Group 3. 207 The similarity at the 5' and 3' ends of the repeat in the different sub-groups of type II-A system 208 and the fact that exchanging leader ends between CRISPR1 and CRISPR3 loci in Sth 209 DGCC771029 impaired adaptation shows that the specificity within the sub-groups of type II-A 210 CRISPR system is most probably attributed by the 3' leader end and not specified by the repeat 211 ends.

\section{Analysis of Cas proteins}


213 We extended our analysis to verify whether the different groups of type II-A CRISPR loci observed

214 based on the 3 ' leader end conservation relates to Cas proteins. The protein sequences of Cas 1

215 belonging to the selected type II-A loci were aligned by MUSCLE and a phylogenetic tree was

216 generated (Fig. 4 and Fig. S3). The loci segregated into 4 main branches, with each branch

217 carrying distinct groups based on the 3' leader end sequence conservation. A sequence

218 alignment of the leader-repeat junction of the different branches show how the Cas 1 sequence is

219 highly correlated with the leader-repeat junction. This confirms previous findings that all the

220 CRISPR-Cas components have coevolved together. ${ }^{46}$ The phylogenetic tree shows that Group 1

221 loci are very distant in lineage, which has later evolved into different subsets with specific leader-

222 repeat-Cas1 combinations. Group 2 and Group 3 evolved for very specific genera, while Group 1

223 has accommodated divergent genera.

224 A similar analysis was done for the Cas2, Csn2, and Cas9 proteins. The sequence alignments

225 generated using the sequences of the corresponding Cas proteins were used to build 226 phylogenetic trees (Fig. 5, 6 and Fig. S4 and S5). All the clades in the different trees have similar

227 3'-leader ends, except for a few differences in the Cas9 phylogenetic tree where some Group 3

228 members appeared along with Group 1. A closer analysis of the sequences showed high

229 variability in the Cas9 lengths, including an extremely short Cas9 sequence (Plo NGRI0510Q) in

230 the outliers, which may have contributed to the random placement of this Cas 9 protein. Cas 9 also

231 showed a branch (1b) for Group 1 that did not show prominent leader end conservation as that

232 was observed in branch 1a. Except for the few differences in Cas9, our results indicate the

233 presence of distinct groups within the type II-A CRISPR systems that possess conserved 3' leader

234 ends and group-specific Cas proteins. It was proposed earlier that the longer version of Csn2

235 evolved first and the shorter Csn2 proteins evolved from the longer versions. ${ }^{56}$ Interestingly, our

236 phylogenetic analysis agrees with this and shows a branch that represents the ancestor with an

237 average Csn2 length of 320 amino acids (Fig. 6). Three main branches evolved from the ancestor

238 and all of them have an average amino acid length of 218-230 amino acids, but varying 3' leader 
239 ends (Table S4). Thus, the ATTTGAG motif is ancestral and universal in the type II-A systems,

240 which later developed to have a similar (ATTTGAG), deviating (CTRCGAG), or less conserved

241 (CG) 3' leader end, with a corresponding change in the protein sequences of all four type II-A Cas

242 proteins. Examining the lengths of Cas1, Cas2, and Cas9 from different groups, we did not

243 observe a strong correlation between the average length of these Cas proteins and the branching

244 group that they belonged.

\section{Discussion}

246 Though previous studies have shown that the leader-repeat region is important for adaptation,

247 the specific features of the leader-repeat region that may recruit Cas1-Cas2 for adaptation are

248 not clearly defined. We focused on the sequence conservation around the leader-repeat junction

249 and found three distinct DNA motifs at the 3' leader ends; Group 1 (ATTTGAG), Group 2

250 (CTRCGAG), and Group 3 (CG). The presence of a conserved 3' leader end, despite a low

251 sequence conservation in the upstream regions of the leader in bacteria belonging to 50 different

252 unrelated genera, strongly suggests that these DNA motifs play a role in site-specific adaptation.

253 One of the most interesting observations from this analysis is the conservation of GAGGTTT as

254 the leader-repeat junction in both Group 1 and Group 2 (82\%, 117 out of 142 loci) of the type II-

255 A system.

256 Several studies have implicated the importance of the leader and repeat sequences to drive

257 faithful adaptation. Terns and coworkers reported that streptococci with repeats similar to that

258 present in the CRISPR1 locus (Group 1) of Sth DGCC7710 have the 3' leader end conserved as

259 ATTTGAG. The accompanying experimental work clearly demonstrated that the 10 nucleotides

260 present at the 3 ' end of the leader and the first repeat are essential and sufficient for adaptation,

261 even in a non-CRISPR locus ${ }^{29}$. It was concluded that sequences at the leader-repeat junction

262 recruits the adaptation machinery to this region for integration of new spacers ${ }^{29}$. In a recent study

263 that analyzed the spacer variation in 126 human isolates of $S$. agalactiae, the 3' leader end of 
264 most of the isolates had a TACGAG sequence ${ }^{66}$. Our analysis that focused on many divergent 265 genera uncovered that the DNA motifs that were previously known to be important for 266 streptococcal adaptation is in fact more ubiquitous and conserved across different bacteria.

267 The importance of the sequences of the leader and the first repeat in driving adaptation is 268 conserved across different CRISPR types. The 60 nucleotides towards the 3' end of the type I-E 269 CRISPR locus of Escherichia coli is essential for adaptation ${ }^{30}$. The disruption of the first repeat 270 sequence that left the stem-loop structure intact prevented successful adaptation in a type IE 271 system leading to the conclusion that the cruciform structure of the repeat alone is not sufficient 272 for adaptation ${ }^{67}$. Another study showed that the -2 (second last position of leader) and +1 (first 273 nucleotide of repeat) positions of leader-repeat regions are crucial for adaptation in E. coli (type $274 \mathrm{IE}$ ) and Sulfolobus solfataricus (type $\mathrm{I}-\mathrm{A}$ ) 68,69 Other studies have experimentally demonstrated that 275 leader and repeat sequences are important for adaptation in streptococcal type II-A systems 276 corresponding to the groups 1 and 2 that we identified in our study ${ }^{29,41}$. Comparing our results 277 with the earlier studies show that leader-repeat sequence conservation that we observed in type 278 II-A sub-groups is relevant for adaptation across diverse bacteria.

279 There is an interplay between the leader and repeat sequences in adaptation that is CRISPR 280 type-specific. For example, in the type I-B system of Haloarcula hispanica, inverted repeats (IR) 281 present within the first repeat are essential for recruiting the adaptation machinery to the leader282 repeat junction. Once the IRs are located within a repeat, a cut is made by the Cas1-Cas2 283 complex at the leader-repeat junction and the sequence of the leader is critical for this step. The 284 second cut at the repeat-spacer end is based on a ruler-mechanism and does not depend on the 285 sequence of the repeat ${ }^{54}$. Whereas in a type II-A system corresponding to our Group 2, it was 286 shown that the repeat-spacer and repeat-leader ends have the same probability of getting cleaved 287 by Cas1-Cas2, but for a faithful adaptation, the leader-repeat junction is essential ${ }^{41}$. In the Group 2881 type II-A locus of Sth DGCC7710, mutations in the last 10 nucleotides of the leader abolished 289 adaptation ${ }^{29}$. This study also elegantly showed that substitution of the 10 nucleotides at the 3 ' end 
290 of Group 1 leader with that of Group 2 leader abolished adaptation following a phage challenge,

291 further emphasizing the importance of the locus specific leader-repeat junction in

292 adaptation ${ }^{29}$. Thus, our observation of the group-specific sequence conservation in type II-A

293 systems at the leader end, along with a lack of distinct group-specific motifs at the 5' and 3' ends

294 of the first repeat, shows that the sub-group specificity in type II-A adaptation arises from the

295 leader sequences that might be specifically distinguished by the Cas1-Cas2 proteins belonging

296 to each sub-group.

297 Both groups 1 and 2 are active for adaptation and interference ${ }^{4,35,40,42,44-47}$, while Group 3 has

298 been shown to be active in DNA interference ${ }^{70}$. Introduction of the type II-A Group 2 locus into $E$.

299 coli protected the bacterium from phage and plasmid infection ${ }^{42}$, demonstrating that intrinsic

300 specificities of protein and DNA components of a CRISPR sub-type are sufficient to drive

301 adaptation and there are no organismal requirements. The three different DNA motifs that we

302 observed at the 3'-end of the leader of the type II-A CRISPR loci may represent three specific

303 functional adaptation units, perhaps guided by leader-sequence specific Cas protein(s). The third

304 group, which consists mostly of lactobacilli, with only two nucleotides conserved instead of seven

305 nucleotides at the 3' leader end in Groups 1 and 2 may represent a more diverse adaptation

306 complex where the protein-DNA sequence interactions are not as tight. It was noted recently that

307 there is considerable variation in the spacer content, even in the ancestral spacers, in L. gasseri

308 strains that indicates considerable divergence between the strains ${ }^{70}$, thus accounting for the low

309 level of sequence conservation at the 3'-end of the leader. This study also showed that the

310 spacers matched plasmids and temperate phages, though it is not clear how L. gasseri acquires

311 spacers from prophages that do not pose threat to bacterial survival ${ }^{70}$. These environmental

312 factors may contribute to the low sequence conservation at the 3'-end of the leader in Group 3.

313 Further experiments will be required to assess the adaptation process in Group 3. Group 3 could

314 also be a result of an insufficient amount of genomic data available to completely resolve any

315 more conserved motifs hidden in the different leader end sequences found within the group. 
316 Repeat sequences are specific to a CRISPR locus, even within sub-types ${ }^{46,56}$. The first two

317 nucleotides of the first repeat was shown to be essential for adaptation in the CRISPR1 locus of

318 Sth $^{29}$ and the first six nucleotides are essential in adaptation in S. pyogenes. ${ }^{41}$ The importance of

$319 \mathrm{G}$ as the first nucleotide in the repeat for efficient disintegration reaction was demonstrated for

320 both $E$. coli and S. solfataricus Cas 1 proteins ${ }^{71}$. We found conservation at the ends of the repeat

321 between groups (Fig. 3). Only 17/167 loci analyzed did not possess a GTTT at the 5'-end of the

322 first repeat, and $3 / 167$ of the loci did not possess a conserved $C$ at the 3 ' end. It was previously

323 reported that purified E. coli Cas1 possesses nuclease activity against several types of DNA

324 substrates including single stranded DNA, replication forks, Holliday junctions etc. without

325 adequate intrinsic sequence specificity and that the four-way DNA junctions recruits Cas1

326 protein $^{72}$. Recently, more studies point to the importance of DNA sequence specificity, especially

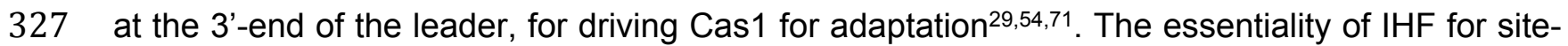

328 specific adaptation in type - indicates that even though Cas1 may have the ability of non-

329 sequence specific cleavage in certain CRISPR types, tight regulation by other cellular proteins

330 may enhance site-specific spacer insertion. The position of the IHF site is 9 to 35 nucleotides

331 upstream of the leader-repeat junction in type I systems ${ }^{55}$. The 20 nucleotides of the 3' leader end

332 that we analyzed for the type II-A did not possess any similarity to the IHF binding site. It is

333 possible that a cruciform structure formed by leader-repeat or repeat palindromic regions along

334 with specific leader-repeat sequences may recruit the Cas1-Cas2 complex for spacer insertion

335 and that this requirement is critical under in vivo conditions.

336 All four Cas proteins are essential for successful adaptation in vivo in type II-A systems ${ }^{4,30,39}$.

337 Previous studies have shown that the CRISPR components and Cas proteins have coevolved ${ }^{46}$.

338 Our analysis showed that all the four type II-A specific Cas proteins and the first repeat clustered

339 into identical groups with similar 3' leader ends. Even though Cas1 protein sequences within type

340 II-A are highly conserved, there are certain differences that segregate them into distinct groups

341 and interestingly these groups have distinct leader sequence conservation. It was previously 
342 reported that type II-A CRISPR systems have distinct operon organization that correlates with

343 Csn2 sequence, making Csn2 the signature protein for type II-A systems ${ }^{56}$. The longer version of

344 Csn2 originated first and the shorter version evolved from the longer version ${ }^{56}$. Our analysis

345 shows that the length of Csn2 is conserved across different clusters (Fig. 5). Looking at Fig. 6,

346 branch 1a segregated early from the rest of the tree and consists of the longer version of Csn2,

347 while branches $1 \mathrm{~b}, 2$, and 3 all consist of the shorter version of Csn2. Correlating Csn2 branching

348 to the leader end sequences, it is evident that our Group 1 motif of ATTTGAG is present in the

349 ancestral strains, which later evolved to distinct sub-groups possessing either Group 1, Group 2

350 (CTRCGAG) or Group 3 (CG) leader ends.

\section{Conclusion}

353 We present an extensive bioinformatic analysis of type II-A CRISPR systems spanning 50 354 different bacterial genera. We demonstrated the ubiquitous nature of two distinct DNA motifs at 355 the 3' end of the leader: Group 1 (ATTTGAG) and Group 2 (CTRCGAG) and also discovered a 356 new group (Group 3) with a limited sequence conservation at the 3'-end of the leader. The leader357 repeat junction is highly conserved for Groups 1 and 2 as GAGGTTT. Our work proposes that the 358 Cas proteins of each sub-group within the type II-A system should make sequence-specific 359 association with its cognate DNA region for successful spacer insertion. The observations further 360 strengthen the previous notion that a highly specific interplay between Cas proteins and cognate 361 leader-repeat regions is essential for effective adaptation $29,30,37,67,68$.

\section{Acknowledgements}

364 We thank Sungho Suh for help with genomic data collection and processing. 


\section{References}

367 1. Marraffini, L.A. \& Sontheimer, E.J. CRISPR interference: RNA-directed adaptive immunity in bacteria and archaea. Nat Rev Genet 11, 181-90 (2010).

370

371

372

373

374

375

376

377

378

379

380

381

382

383

384

385

386

387

388

389

390

391

392

393

394

395

396

397

398

399

400

401

402

403

404

405

406

407

2. Sorek, R., Lawrence, C.M. \& Wiedenheft, B. CRISPR-mediated adaptive immune systems in bacteria and archaea. Annu Rev Biochem 82, 237-66 (2013).

3. Mojica, F.J., Diez-Villasenor, C., Garcia-Martinez, J. \& Soria, E. Intervening sequences of regularly spaced prokaryotic repeats derive from foreign genetic elements. $J \mathrm{Mol}$ Evol 60, 174-82 (2005).

4. Barrangou, R., Fremaux, C., Deveau, H., Richards, M., Boyaval, P., Moineau, S., Romero, D.A. \& Horvath, P. CRISPR provides acquired resistance against viruses in prokaryotes. Science 315, 1709-12 (2007).

5. Makarova, K.S., Grishin, N.V., Shabalina, S.A., Wolf, Y.I. \& Koonin, E.V. A putative RNAinterference-based immune system in prokaryotes: computational analysis of the predicted enzymatic machinery, functional analogies with eukaryotic RNAi, and hypothetical mechanisms of action. Biol Direct 1, 7 (2006).

6. Marraffini, L.A. \& Sontheimer, E.J. Invasive DNA, chopped and in the CRISPR. Structure 17, 786-8 (2009).

7. Marraffini, L.A. \& Sontheimer, E.J. Self versus non-self discrimination during CRISPR RNA-directed immunity. Nature 463, 568-71 (2010).

8. Marraffini, L.A. \& Sontheimer, E.J. CRISPR interference limits horizontal gene transfer in staphylococci by targeting DNA. Science 322, 1843-5 (2008).

9. Brouns, S.J., Jore, M.M., Lundgren, M., Westra, E.R., Slijkhuis, R.J., Snijders, A.P., Dickman, M.J., Makarova, K.S., Koonin, E.V. \& van der Oost, J. Small CRISPR RNAs guide antiviral defense in prokaryotes. Science 321, 960-4 (2008).

10. Hale, C.R., Zhao, P., Olson, S., Duff, M.O., Graveley, B.R., Wells, L., Terns, R.M. \& Terns, M.P. RNA-guided RNA cleavage by a CRISPR RNA-Cas protein complex. Cell 139, 945-56 (2009).

11. Abudayyeh, O.O., Gootenberg, J.S., Konermann, S., Joung, J., Slaymaker, I.M., Cox, D.B., Shmakov, S., Makarova, K.S., Semenova, E., Minakhin, L., Severinov, K., Regev, A., Lander, E.S., Koonin, E.V. \& Zhang, F. C2c2 is a single-component programmable RNA-guided RNA-targeting CRISPR effector. Science (2016).

12. Makarova, K.S. \& Koonin, E.V. Annotation and Classification of CRISPR-Cas Systems. Methods Mol Biol 1311, 47-75 (2015).

13. Makarova, K.S., Wolf, Y.I., Alkhnbashi, O.S., Costa, F., Shah, S.A., Saunders, S.J., Barrangou, R., Brouns, S.J.J., Charpentier, E., Haft, D.H., Horvath, P., Moineau, S., Mojica, F.J.M., Terns, R.M., Terns, M.P., White, M.F., Yakunin, A.F., Garrett, R.A., van der Oost, J., Backofen, R. \& Koonin, E.V. An updated evolutionary classification of CRISPR-Cas systems. Nat Rev Micro 13, $722-736$ (2015).

14. Jackson, R.N. \& Wiedenheft, B. A Conserved Structural Chassis for Mounting Versatile CRISPR RNA-Guided Immune Responses. Mol Cell 58, $722-8$ (2015).

15. Reeks, J., Naismith, J.H. \& White, M.F. CRISPR interference: a structural perspective. Biochem J 453, 155-66 (2013). 
408 16. Makarova, K.S., Aravind, L., Wolf, Y.I. \& Koonin, E.V. Unification of Cas protein

409

410

411

412

413

414

415

416

417

418

419

420

421

422

423

424

425

426

427

428

429

430

431

432

433

434

435

436

437

438

439

440

441

442

443

444

445

446

447

448

449

450

451

452 families and a simple scenario for the origin and evolution of CRISPR-Cas systems. Biol Direct 6, 38 (2011).

17. Koonin, E.V. \& Makarova, K.S. CRISPR-Cas: evolution of an RNA-based adaptive immunity system in prokaryotes. RNA Biol 10, 679-86 (2013).

18. Shmakov, S., Abudayyeh, O.O., Makarova, K.S., Wolf, Y.I., Gootenberg, J.S., Semenova, E., Minakhin, L., Joung, J., Konermann, S., Severinov, K., Zhang, F. \& Koonin, E.V. Discovery and Functional Characterization of Diverse Class 2 CRISPR-Cas Systems. Mol Cell (2015).

19. Jinek, M., Chylinski, K., Fonfara, I., Hauer, M., Doudna, J.A. \& Charpentier, E. A programmable dual-RNA-guided DNA endonuclease in adaptive bacterial immunity. Science 337, 816-21 (2012).

20. Jinek, M., Jiang, F., Taylor, D.W., Sternberg, S.H., Kaya, E., Ma, E., Anders, C., Hauer, M., Zhou, K., Lin, S., Kaplan, M., Iavarone, A.T., Charpentier, E., Nogales, E. \& Doudna, J.A. Structures of Cas 9 endonucleases reveal RNA-mediated conformational activation. Science 343, 1247997 (2014).

21. Nishimasu, H., Ran, F.A., Hsu, P.D., Konermann, S., Shehata, S.I., Dohmae, N., Ishitani, R., Zhang, F. \& Nureki, O. Crystal structure of Cas9 in complex with guide RNA and target DNA. Cell 156, 935-49 (2014).

22. Zetsche, B., Gootenberg, J.S., Abudayyeh, O.O., Slaymaker, I.M., Makarova, K.S., Essletzbichler, P., Volz, S.E., Joung, J., van der Oost, J., Regev, A., Koonin, E.V. \& Zhang, F. Cpf1 is a single RNA-guided endonuclease of a class 2 CRISPR-Cas system. Cell 163, 759-71 (2015).

23. Sternberg, S.H. \& Doudna, J.A. Expanding the Biologist's Toolkit with CRISPR-Cas9. Mol Cell 58, 568-574 (2015).

24. Sontheimer, E.J. \& Barrangou, R. The Bacterial Origins of the CRISPR GenomeEditing Revolution. Hum Gene Ther 26, 413-24 (2015).

25. Ishino, Y., Shinagawa, H., Makino, K., Amemura, M. \& Nakata, A. Nucleotide sequence of the iap gene, responsible for alkaline phosphatase isozyme conversion in Escherichia coli, and identification of the gene product. J Bacteriol 169, 5429-33 (1987).

26. Jansen, R., Embden, J.D., Gaastra, W. \& Schouls, L.M. Identification of genes that are associated with DNA repeats in prokaryotes. Mol Microbiol 43, 1565-75 (2002).

27. Haft, D.H., Selengut J Fau - Mongodin, E.F., Mongodin Ef Fau - Nelson, K.E. \& Nelson, K.E. A guild of 45 CRISPR-associated (Cas) protein families and multiple CRISPR/Cas subtypes exist in prokaryotic genomes. PLoS Comput Biol (2005).

28. Pougach, K., Semenova E Fau - Bogdanova, E., Bogdanova E Fau - Datsenko, K.A., Datsenko Ka Fau - Djordjevic, M., Djordjevic M Fau - Wanner, B.L., Wanner Bl Fau Severinov, K. \& Severinov, K. Transcription, processing and function of CRISPR cassettes in Escherichia coli. (2010).

29. Wei, Y., Chesne, M.T., Terns, R.M. \& Terns, M.P. Sequences spanning the leaderrepeat junction mediate CRISPR adaptation to phage in Streptococcus thermophilus. Nucleic Acids Res 43, 1749-58 (2015).

30. Yosef, I., Goren, M.G. \& Qimron, U. Proteins and DNA elements essential for the CRISPR adaptation process in Escherichia coli. Nucleic Acids Res 40, 5569-76 (2012). 
453 31. Mojica, F.J., Diez-Villasenor, C., Garcia-Martinez, J. \& Almendros, C. Short motif sequences determine the targets of the prokaryotic CRISPR defence system.

455

456

457

458

459

460

461

462

463

464

465

466

467

468

469

470

471

472

473

474

475

476

477

478

479

480

481

482

483

484

485

486

487

488

489

490

491

492

493

494

495

496

497

Microbiology 155, 733-40 (2009).

32. Shah, S.A., Erdmann, S., Mojica, F.J. \& Garrett, R.A. Protospacer recognition motifs: mixed identities and functional diversity. RNA Biol 10, 891-9 (2013).

33. Amitai, G. \& Sorek, R. CRISPR-Cas adaptation: insights into the mechanism of action. Nat Rev Microbiol 14, 67-76 (2016).

34. Nunez, J.K., Kranzusch, P.J., Noeske, J., Wright, A.V., Davies, C.W. \& Doudna, J.A. Cas1Cas2 complex formation mediates spacer acquisition during CRISPR-Cas adaptive immunity. Nat Struct Mol Biol 21, 528-34 (2014).

35. Garneau, J.E., Dupuis, M.E., Villion, M., Romero, D.A., Barrangou, R., Boyaval, P., Fremaux, C., Horvath, P., Magadan, A.H. \& Moineau, S. The CRISPR/Cas bacterial immune system cleaves bacteriophage and plasmid DNA. Nature 468, 67-71 (2010).

36. Erdmann, S. \& Garrett, R.A. Selective and hyperactive uptake of foreign DNA by adaptive immune systems of an archaeon via two distinct mechanisms. Mol Microbiol 85, 1044-56 (2012).

37. Diez-Villasenor, C., Guzman, N.M., Almendros, C., Garcia-Martinez, J. \& Mojica, F.J. CRISPR-spacer integration reporter plasmids reveal distinct genuine acquisition specificities among CRISPR-Cas I-E variants of Escherichia coli. RNA Biol 10, 792802 (2013).

38. Wei, Y., Terns, R.M. \& Terns, M.P. Cas9 function and host genome sampling in Type II-A CRISPR-Cas adaptation. Genes Dev 29, 356-61 (2015).

39. Heler, R., Samai, P., Modell, J.W., Weiner, C., Goldberg, G.W., Bikard, D. \& Marraffini, L.A. Cas9 specifies functional viral targets during CRISPR-Cas adaptation. Nature 519, 199-202 (2015).

40. Paez-Espino, D., Morovic, W., Sun, C.L., Thomas, B.C., Ueda, K., Stahl, B., Barrangou, R. \& Banfield, J.F. Strong bias in the bacterial CRISPR elements that confer immunity to phage. Nat Commun 4, 1430 (2013).

41. Wright, A.V. \& Doudna, J.A. Protecting genome integrity during CRISPR immune adaptation. LID - 10.1038/nsmb.3289 [doi]. Nat Struct Mol Biol (2016).

42. Sapranauskas, R., Gasiunas, G., Fremaux, C., Barrangou, R., Horvath, P. \& Siksnys, V. The Streptococcus thermophilus CRISPR/Cas system provides immunity in Escherichia coli. Nucleic Acids Res 39, 9275-82 (2011).

43. Horvath, P. \& Barrangou, R. CRISPR/Cas, the immune system of bacteria and archaea. Science 327, 167-70 (2010).

44. Carte, J., Christopher, R.T., Smith, J.T., Olson, S., Barrangou, R., Moineau, S., Glover, C.V., 3rd, Graveley, B.R., Terns, R.M. \& Terns, M.P. The three major types of CRISPRCas systems function independently in CRISPR RNA biogenesis in Streptococcus thermophilus. Mol Microbiol 93, 98-112 (2014).

45. Deveau, H., Barrangou, R., Garneau, J.E., Labonte, J., Fremaux, C., Boyaval, P., Romero, D.A., Horvath, P. \& Moineau, S. Phage response to CRISPR-encoded resistance in Streptococcus thermophilus. J Bacteriol 190, 1390-400 (2008).

46. Horvath, P., Romero, D.A., Coute-Monvoisin, A.C., Richards, M., Deveau, H., Moineau, S., Boyaval, P., Fremaux, C. \& Barrangou, R. Diversity, activity, and evolution of CRISPR loci in Streptococcus thermophilus. J Bacteriol 190, 1401-12 (2008). 
498

499

500

501

502

503

504

505

506

507

508

509

510

511

512

513

514

515

516

517

518

519

520

521

522

523

524

525

526

527

528

529

530

531

532

533

534

535

536

537

538

539

540

541

542

543
47. Lopez-Sanchez, M.J., Sauvage, E., Da Cunha, V., Clermont, D., Ratsima Hariniaina, E., Gonzalez-Zorn, B., Poyart, C., Rosinski-Chupin, I. \& Glaser, P. The highly dynamic CRISPR1 system of Streptococcus agalactiae controls the diversity of its mobilome. Mol Microbiol 85, 1057-71 (2012).

48. Yosef, I., Shitrit, D., Goren, M.G., Burstein, D., Pupko, T. \& Qimron, U. DNA motifs determining the efficiency of adaptation into the Escherichia coli CRISPR array. Proc Natl Acad Sci U S A 110, 14396-401 (2013).

49. Jansen, R., van Embden, J.D., Gaastra, W. \& Schouls, L.M. Identification of a novel family of sequence repeats among prokaryotes. OMICS 6, 23-33 (2002).

50. Li, M., Wang, R., Zhao, D. \& Xiang, H. Adaptation of the Haloarcula hispanica CRISPRCas system to a purified virus strictly requires a priming process. Nucleic Acids Res 42, 2483-92 (2014).

51. Lillestol, R.K., Shah, S.A., Brugger, K., Redder, P., Phan, H., Christiansen, J. \& Garrett, R.A. CRISPR families of the crenarchaeal genus Sulfolobus: bidirectional transcription and dynamic properties. Mol Microbiol 72, 259-72 (2009).

52. Bernick, D.L., Cox, C.L., Dennis, P.P. \& Lowe, T.M. Comparative genomic and transcriptional analyses of CRISPR systems across the genus Pyrobaculum. Front Microbiol 3, 251 (2012).

53. Erdmann, S., Le Moine Bauer, S. \& Garrett, R.A. Inter-viral conflicts that exploit host CRISPR immune systems of Sulfolobus. Mol Microbiol 91, 900-17 (2014).

54. Wang, R., Li, M., Gong, L., Hu, S. \& Xiang, H. DNA motifs determining the accuracy of repeat duplication during CRISPR adaptation in Haloarcula hispanica. Nucleic Acids Res (2016).

55. Nunez, J.K., Bai, L., Harrington, L.B., Hinder, T.L. \& Doudna, J.A. CRISPR Immunological Memory Requires a Host Factor for Specificity. Mol Cell (2016).

56. Chylinski, K., Makarova, K.S., Charpentier, E. \& Koonin, E.V. Classification and evolution of type II CRISPR-Cas systems. Nucleic Acids Res 42, 6091-105 (2014).

57. Fonfara, I., Le Rhun, A., Chylinski, K., Makarova, K.S., Lecrivain, A.L., Bzdrenga, J., Koonin, E.V. \& Charpentier, E. Phylogeny of Cas9 determines functional exchangeability of dual-RNA and Cas 9 among orthologous type II CRISPR-Cas systems. Nucleic Acids Res 42, 2577-90 (2014).

58. Boratyn, G.M., Schaffer Aa Fau - Agarwala, R., Agarwala R Fau - Altschul, S.F., Altschul Sf Fau - Lipman, D.J., Lipman Dj Fau - Madden, T.L. \& Madden, T.L. Domain enhanced lookup time accelerated BLAST. Biol Direct (2012).

59. Grissa, I., Vergnaud, G. \& Pourcel, C. The CRISPRdb database and tools to display CRISPRs and to generate dictionaries of spacers and repeats. BMC Bioinformatics $\mathbf{8}$, 172 (2007).

60. Alkhnbashi, O.S., Shah, S.A., Garrett, R.A., Saunders, S.J., Costa, F. \& Backofen, R. Characterizing leader sequences of CRISPR loci. Bioinformatics (2016).

61. Biswas, A., Staals, R.H., Morales, S.E., Fineran, P.C. \& Brown, C.M. CRISPRDetect: A flexible algorithm to define CRISPR arrays. BMC Genomics (2016).

62. Edgar, R.C. MUSCLE: multiple sequence alignment with high accuracy and high throughput. Nucleic Acids Res 32, 1792-7 (2004).

63. Tamura, K., Stecher, G., Peterson, D., Filipski, A. \& Kumar, S. MEGA6: Molecular Evolutionary Genetics Analysis Version 6.0. Molecular Biology and Evolution 30, 2725-2729 (2013). 
544 64. Okonechnikov, K., Golosova O Fau - Fursov, M. \& Fursov, M. Unipro UGENE: a unified 545 bioinformatics toolkit. Bioinformatics 8(2012).

546 65. Crooks, G.E., Hon, G., Chandonia, J.M. \& Brenner, S.E. WebLogo: a sequence logo

547 generator. Genome Res 14, 1188-90 (2004).

548 66. Lier, C., Baticle, E., Horvath, P., Haguenoer, E., Valentin, A.-S., Glaser, P., Mereghetti, L.

549 \& Lanotte, P. Analysis of the type II-A CRISPR-Cas system of Streptococcus agalactiae

550

551

552

553

554

555

556

557

558

559 reveals distinctive features according to genetic lineages. Frontiers in Genetics 6, 214 (2015).

67. Arslan, Z., Hermanns, V., Wurm, R., Wagner, R. \& Pul, U. Detection and characterization of spacer integration intermediates in type I-E CRISPR-Cas system. Nucleic Acids Res 42, 7884-93 (2014).

68. Rollie, C., Schneider, S., Brinkmann, A.S., Bolt, E.L. \& White, M.F. Intrinsic sequence specificity of the Cas1 integrase directs new spacer acquisition. Elife 4(2015).

69. Nunez, J.K., Lee, A.S., Engelman, A. \& Doudna, J.A. Integrase-mediated spacer acquisition during CRISPR-Cas adaptive immunity. Nature 519, 193-8 (2015).

70. Sanozky-Dawes, R., Selle, K., O'Flaherty, S., Klaenhammer, T. \& Barrangou, R.

560 Occurrence and activity of a type II CRISPR-Cas system in Lactobacillus gasseri.

$561 \quad$ Microbiology (2015).

562 71. Rollie, C., Schneider, S., Brinkmann, A.S., Bolt, E.L. \& White, M.F.A.-O.h.o.o. Intrinsic

563

564

565

566

567

568

569

570 sequence specificity of the Cas 1 integrase directs new spacer acquisition. LID 10.7554/eLife.08716 [doi]. Elife (2015).

72. Babu, M., Beloglazova, N., Flick, R., Graham, C., Skarina, T., Nocek, B., Gagarinova, A., Pogoutse, O., Brown, G., Binkowski, A., Phanse, S., Joachimiak, A., Koonin, E.V., Savchenko, A., Emili, A., Greenblatt, J., Edwards, A.M. \& Yakunin, A.F. A dual function of the CRISPR-Cas system in bacterial antivirus immunity and DNA repair. Mol Microbiol 79, 484-502 (2011). 


\section{Figure 1}

Figure 1

Sequence alignment of the last 20 nucleotides of the 3 ' end of the leader and the first repeat of selected Group 1 species. Height of the letters in the WebLogo indicates the degree of conservation at specific nucleotide locations. The leader-repeat end is conserved as 5'ATTTGAGGTTT-3'. 
Smo_F0204

Vat_ACS-134-V-Col7a

Pal_ATCC23263

Dlo_AGR2136

Lba_NK4A179

Asp_D21

Ssp_CAG:314

Fal_ATCC 35896

Fma_ATCC29328

HSU_DSM17243

Rla_ATCC29176

PdU_ATCCBAA-1640

BCa_FSLF6-1037

SPS_ED99

CMi_DSM15897

Fnu_3_1_36A2

Rsp_CAG:197

KVi_DSM20405

Fba_M10-2

Gbe_ATCC700627

Fho_CCUG36813

Sma_ACA-DC198_1

San_C1051_4

Sth_LMD-9_2

Sgo_CH1_2

Ssa_JIM8777_3

Sth_DGCC7710_1

Csp_CAG:230

Bfi_16/4

Bpe_CAG:437

Ere_ATCC33656

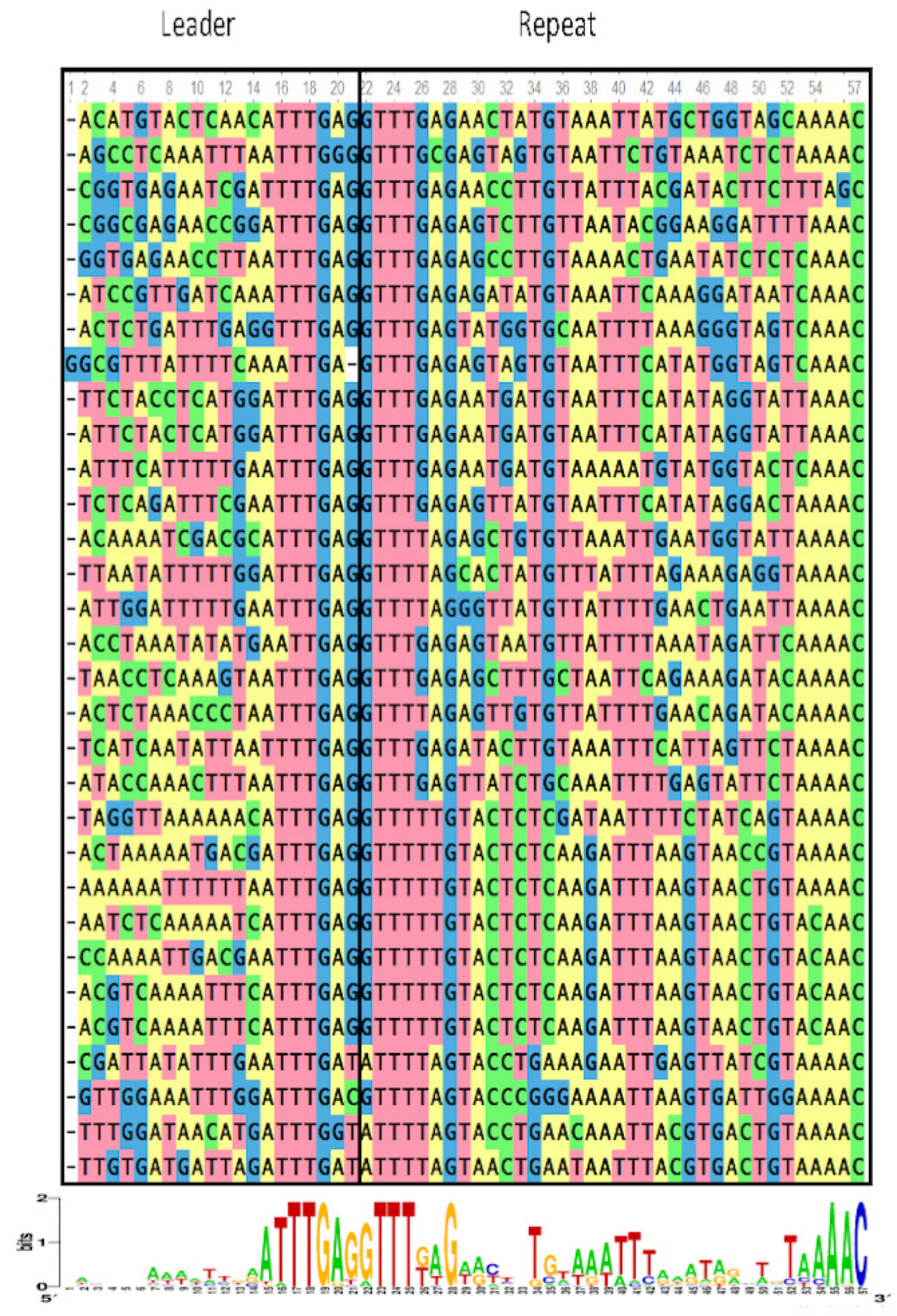




\section{Figure 2}

Figure 2

Sequence alignment of the last 20 nucleotides at the 3 '-end of the leader and the first repeat of selected Group 2(A) and Group 3(B) species. Height of the letters in the WebLogo indicates the degree of conservation at specific nucleotide locations. The leader-repeat of Group 2 loci is conserved as CTRCGAGGTTT, where R represents a purine base. For Group 3 members, this region is conserved as CGGTTT. 
Efa_D32_1

Lmo_10403s_3

Lmo_J0161_4

Lmo_R2-502

EPh_ATCCBAA-412

Emu_QU25_DNA

Spy_A20_1

$S m u \_L J 23 \_2$

Sth_DGCC7710_3

Sth_LMD-9_5

Sag_A909_1

Ssa_SK49

B

$\angle C a \_\angle C 10$

Lfa_KCTC3681

Lsa_TMW1. 1304

LCO_KCTC3167

LrU_ATCC25644

Lje_27-2-CHN

Lga_JV-VO3

Lfe_ATCC14931

Wha_FBL 4

$\angle B U \_N R R L B-30929$

PaC_D3

P7O_NGRIO510Q

LSa_UCC118

Ffr_KCTC3544

Bbi_s17

Lge_KCTC 3527

Oki_DSM_17330
Leader

Repeat

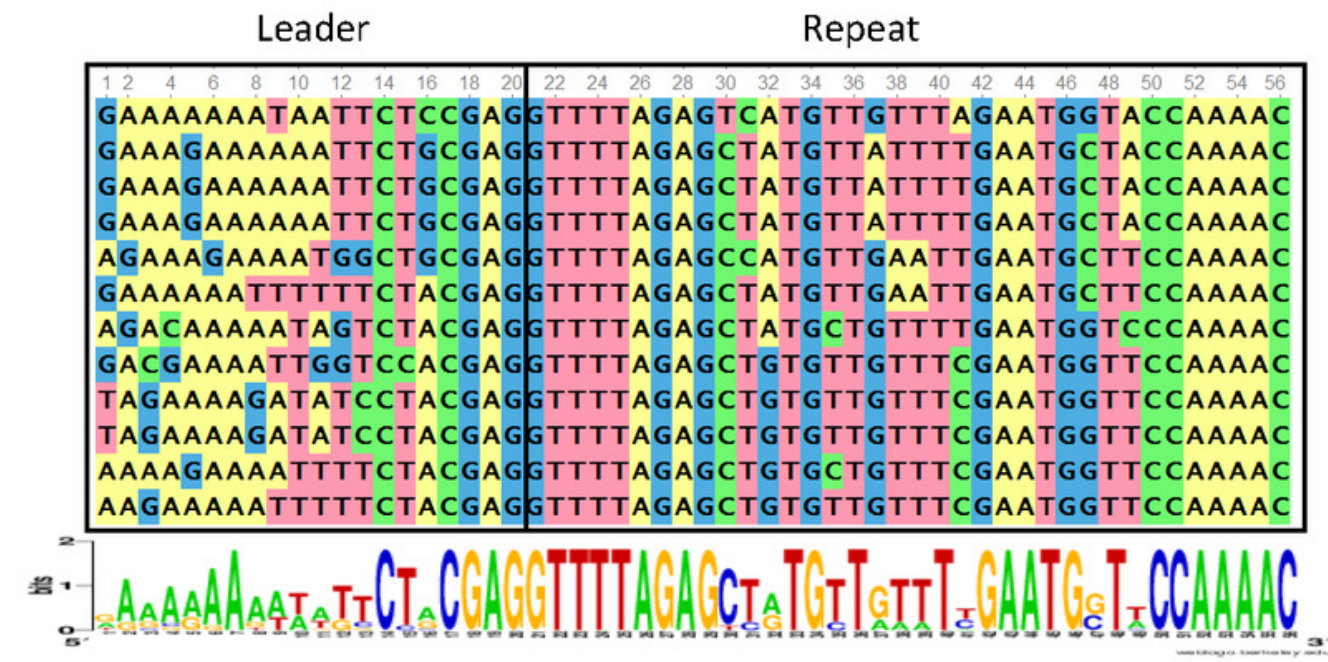

Leader

Repeat

\begin{tabular}{|c|c|}
\hline & \\
\hline & \\
\hline AAGTTGATTAGAAAAC & GTTTTAGAAGTATGTCTTTCTATTTACTT/ \\
\hline & \\
\hline GAAGATAATGTGGAAATAAC & GTTTTAGAAGAGTGTTAAATCAATGAGTTT \\
\hline -CTGTTGATTAAAAAGAGAC & GTTTCAGCTGGATGTCATATCAATGATGTTATGAAD \\
\hline -AAGCTGATGATAAAACGT & GTTTTAGAAGGTTGTTAAATCAGTAAGTTG \\
\hline -АAACTGATTATAAAATTTC & GTTTTAGATGGTTGTTAGATCAATAAGGTTTAGATD \\
\hline -TTTTTGATGAGAAAATAACG & GTCTTGGATGAGTGTCAGATCAGTAGTTCCGAGTAC \\
\hline -TTTTTTATGAGAAATTAA & GTTTCAGAAGAGTGTTAAATCAATAAGTTCAAGTAD \\
\hline -AATTCGGTTATAAAAAATC & GTTTTAGAAG GATGTTAAATCAATAAGGTTAAACC \\
\hline - ATTTTAGTTAGAAAATAAC & GTTTCAGAAGGATGTTAAATCAATAAGGTTAA \\
\hline -ATTTTAGTTAGAAAATA & GTTTCAGAAGGATGTTAAATCAATAAGGTTAAC \\
\hline - АTTTTGATTATAAAGTAAC & GTTTCAGAAGTATGTTAAATCAATAAGGTTAA \\
\hline - СAAAAATCATTGGTCTATC & GCTTTAGATGTATGTCGGATTAATGGGGTTTCT \\
\hline -AGGAATCCTTAAGGCTATC & GTTTCAGATGCCTGTCAGATCAATGACTTTGA \\
\hline ;GCGGTC & GCTTCAGATGTGTGTCAGATCAATGAGGTT \\
\hline & \\
\hline
\end{tabular}

2. 


\section{Figure 3}

Figure 3

Phylogenetic tree generated from the sequence alignment of the first repeats from selected type II-A species. Groups based on the segregation of the Cas1 tree are shown in cyan (Group 1), red (Group 2), and yellow (Group 3). The tree segregates into 6 main clades and WebLogos were produced with alignments of the last 20 nucleotides at the 3 '-end of the leader and the first repeat from the loci within each corresponding branch. 


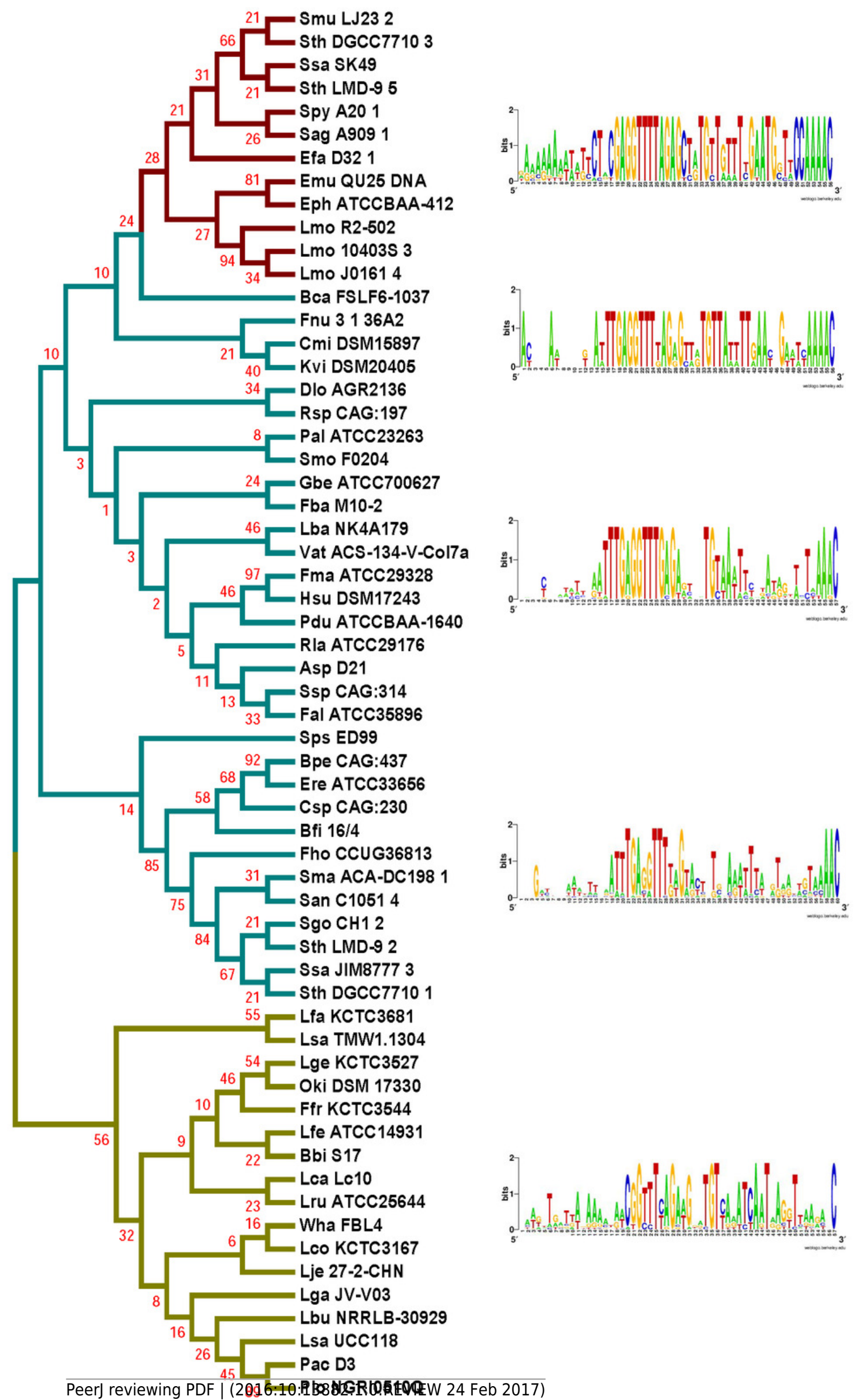

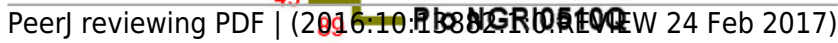




\section{Figure 4}

Figure 4

Phylogenetic tree generated from the sequence alignment of Cas1. Groups are shown in cyan (Group 1), red (Group 2), and yellow (Group 3). WebLogos were generated by aligning the last 7 nucleotides of the leader and the first 4 nucleotides of the repeat from the loci within each corresponding branch. The tree segregates into 4 branches, two branches showing the Group 1 leader end motif, one branch showing the Group 2 motif, and one branch showing the less-conserved Group 3 leader end. Sps ED99 segregated independently from the final branch but was used in the final branch WebLogo construction based on the leader end and protein length. 


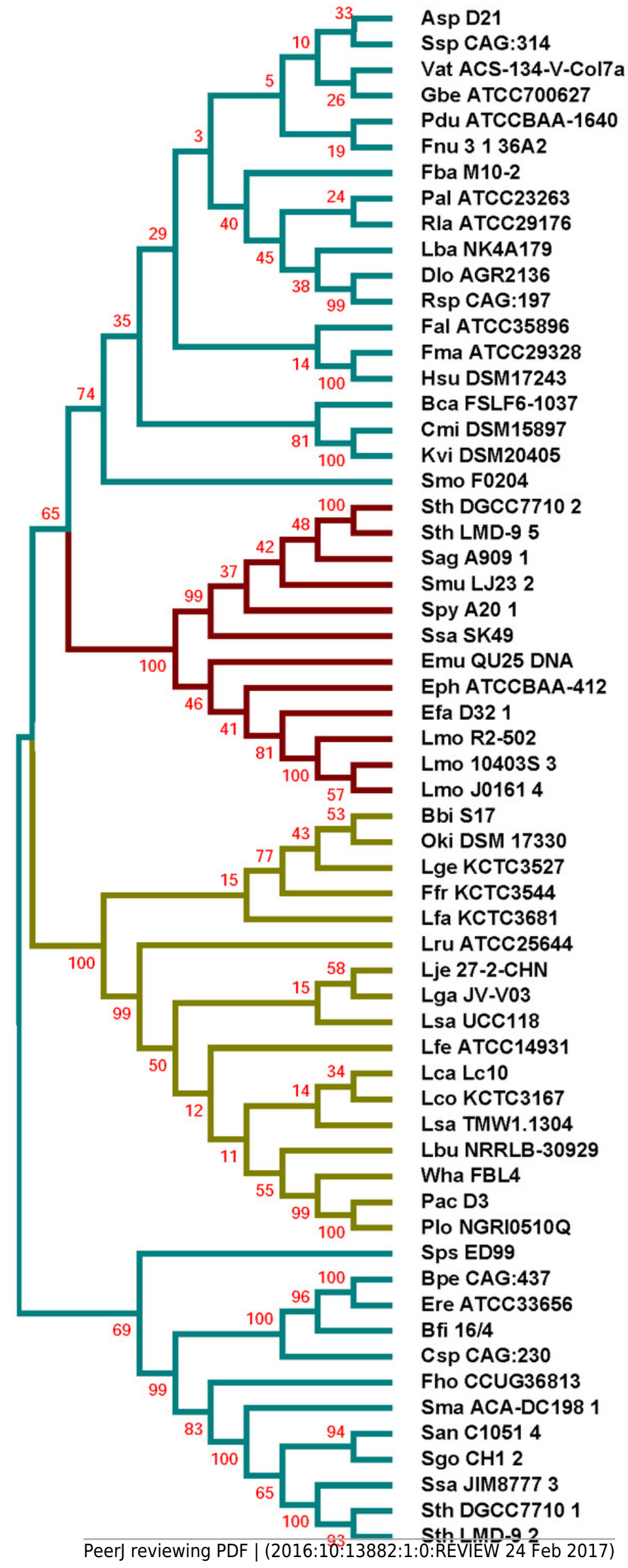

Branch 1b

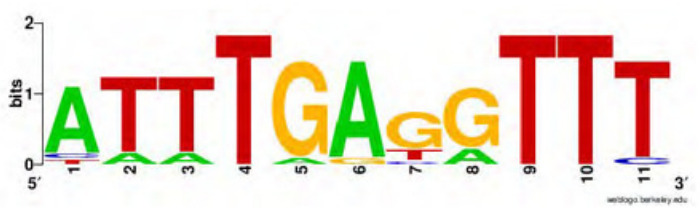

Branch 2

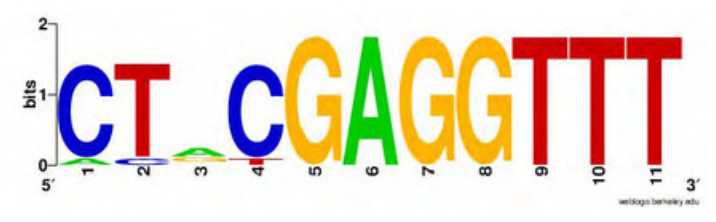

Branch 3

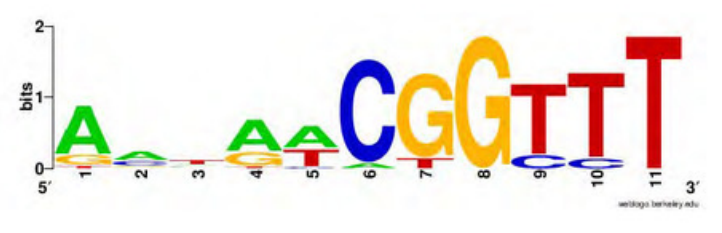

Branch 1a

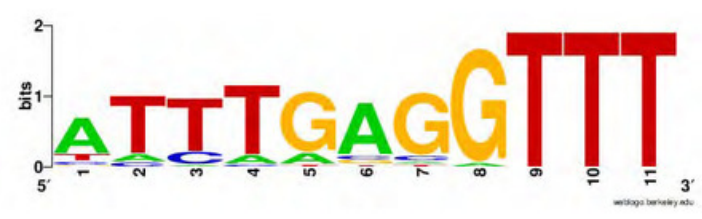




\section{Figure 5}

Figure 5

(A) Phylogenetic tree generated from the sequence alignment of Cas9. Groups based on the segregation of the Cas1 tree are shown in cyan (Group 1), red (Group 2), and yellow (Group 3). The tree shows 5 different branches with two branches showing the Group 1 leader end motif, one branch showing the Group 2 motif, and one branch representing the lessconserved Group 3 leader end. One of the branches represent a very loosely conserved Group 1 loci. Three members of Group 3 segregated away from the normal cluster, of which Plo NGRI0510Q has a very short Cas9 sequence. Lru ATCC25644 and Lfa KCTC3681 have normal length Cas9 sequences. (B) Phylogenetic tree generated from the sequence alignment of Cas2. All the four branches segregate similarly to those of Cas1 phylogenetic tree. WebLogos for both panels of the figure were generated by aligning the last 7 nucleotides of the leader and the first 4 nucleotides of the repeat from the loci within each corresponding branch. 
A

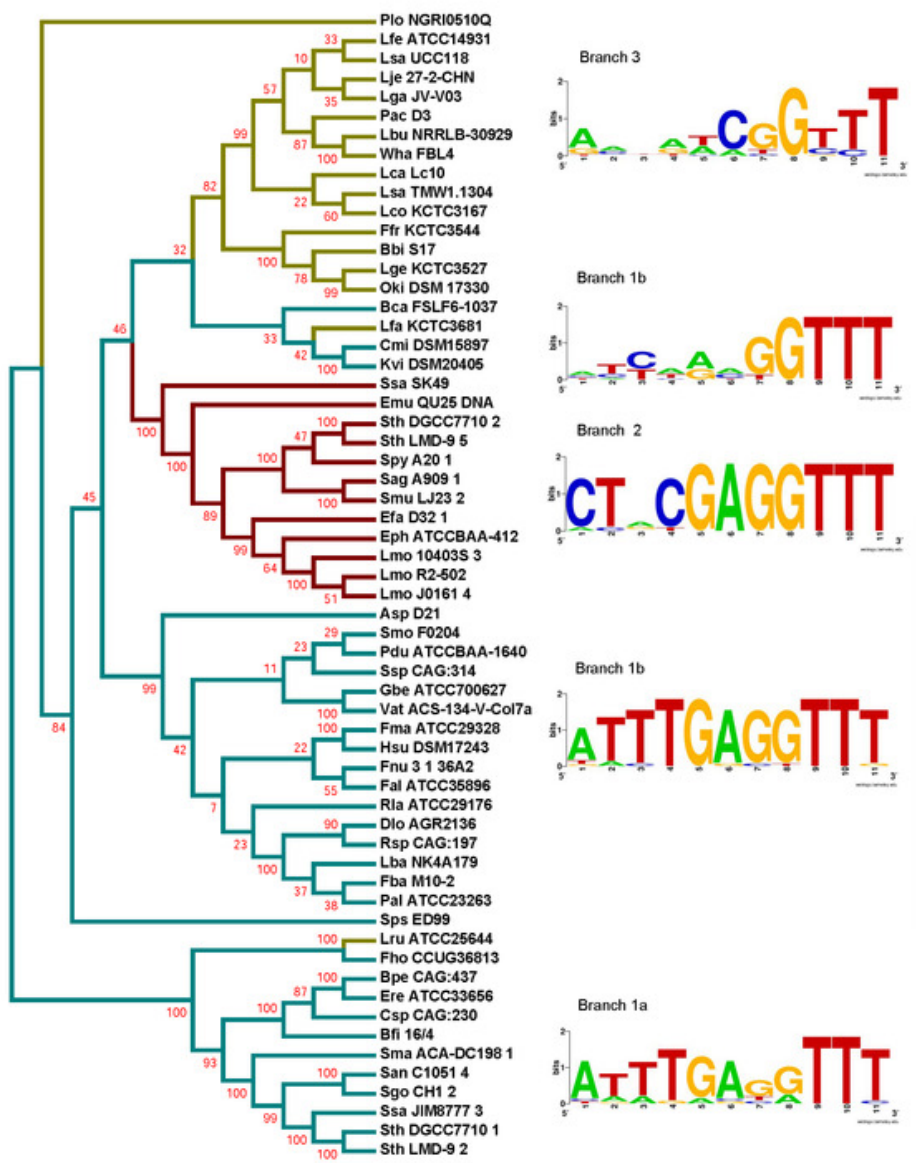

B

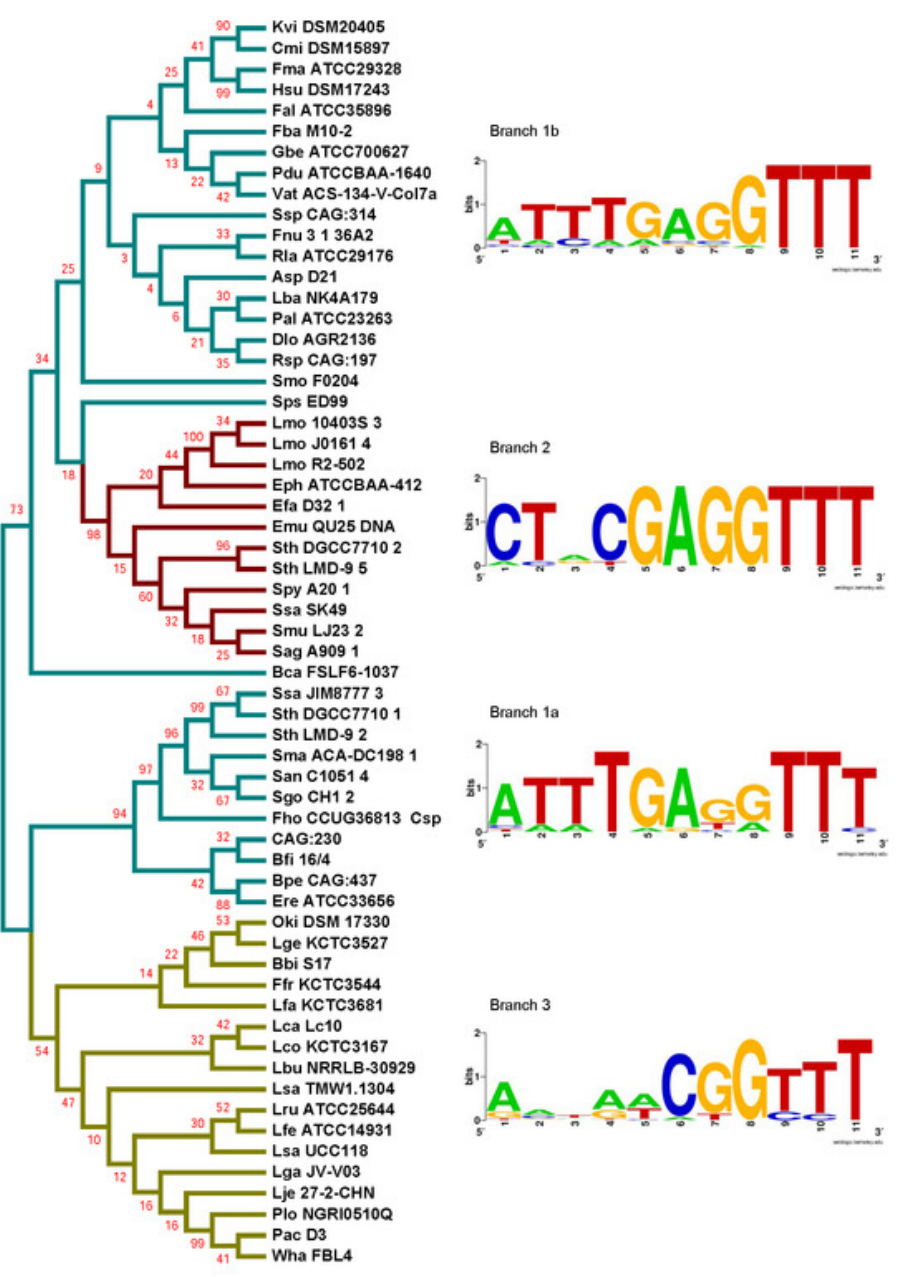




\section{Figure 6}

Figure 6

Phylogenetic tree generated from the sequence alignment of Csn2. Groups based on the segregation of the Cas1 tree are shown in cyan (Group 1), red (Group 2), and yellow (Group 3). WebLogos were generated from aligning the last 7 nucleotides of the leader and the first 4 nucleotides of the repeat from the loci within each corresponding branch. Values next to branch labels indicate the average length of the proteins (in amino acids, aa) within the branch. Two branches show the Group 1 leader end motif, one branch shows the Group 2 motif, and one branch shows the less conserved Group 3 leader end. 

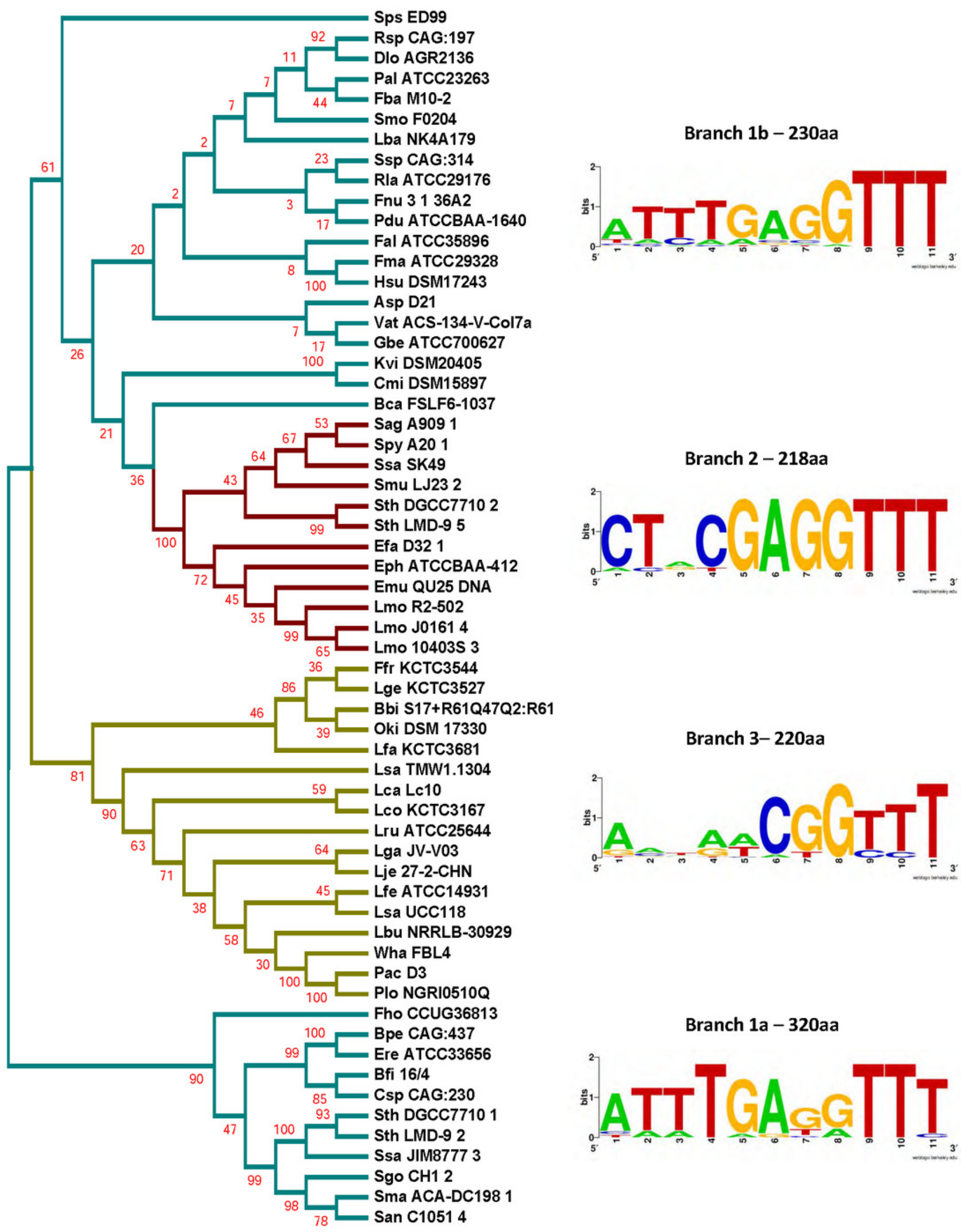

Branch 2 - 218aa

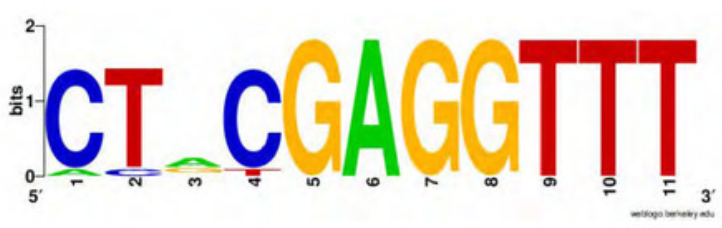

Branch 3- 220aa

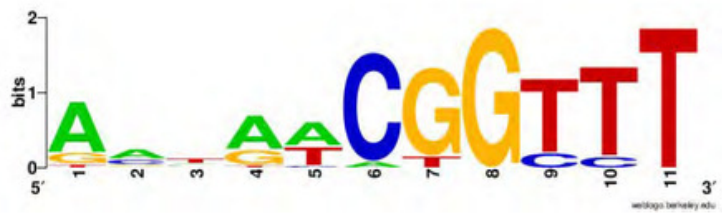

Branch 1a - 320aa

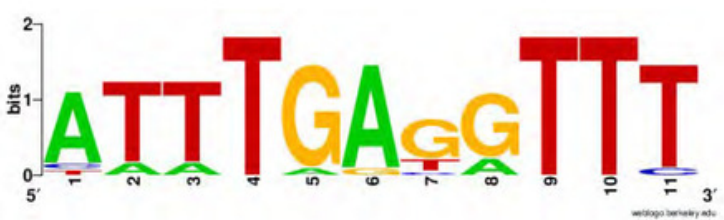

